\title{
CASAS INTELIGENTES: O QUE ELAS PODEM OFERECER PARA IDOSOS
}

\author{
Gabriel Amaral Costas 1 \\ Gabriel Aparecido Zucoloto \\ Vinício Fragoso Mendes ${ }^{3}$ \\ Flávio Pavesi Simão 4
}

Resumo: Este artigo busca identificar os maiores problemas enfrentados pela população idosa em suas casas, e sugerir alternativas existentes no mercado que seriam interessantes para resolução destes problemas. Para isso, foi aplicado um questionário para idosos residentes na cidade de Jerônimo Monteiro - ES, com idades entre 60 a 80 anos. Os resultados se mostraram promissores principalmente pelo fato de $88,2 \%$ dos entrevistados não terem medo das novas tecnologias, não possuindo, apenas, o acesso a elas. Por fim, pode-se concluir que as novas tecnologias para casas inteligentes podem oferecer para pessoas idosas uma maior qualidade de vida e mais comodidade do que em suas residências atuais.

Palavras-chave: Automação; Casas inteligentes; Tecnologia; Idosos.

\footnotetext{
${ }^{1}$ Tecnologia em Análise e Desenvolvimento de Sistemas/Instituto Federal do Espírito Santo - Campus de Alegre, Brasil. E-mail: gabriel_costa_19@hotmail.com.

${ }^{2}$ Tecnologia em Análise e Desenvolvimento de Sistemas/Instituto Federal do Espírito Santo - Campus de Alegre, Brasil. E-mail: bielaparecidozucoloto@gmail.com.

${ }^{3}$ Tecnologia em Análise e Desenvolvimento de Sistemas/Instituto Federal do Espírito Santo - Campus de Alegre, Brasil. E-mail: vinicio0408@gmail.com.

${ }^{4}$ Tecnologia em Análise e Desenvolvimento de Sistemas/Instituto Federal do Espírito Santo - Campus de Alegre, Brasil. E-mail: fpsimao@ifes.edu.br.
} 